\title{
Concurrent use of long-acting bronchodilators in COPD and the risk of adverse cardiovascular events
}

\author{
Samy Suissa, Sophie Dell'Aniello and Pierre Ernst \\ Affiliation: Centre for Clinical Epidemiology, Lady Davis Institute - Jewish General Hospital, Dept of Epidemiology \\ and Biostatistics, McGill University, Montréal, QC, Canada. \\ Correspondence: Samy Suissa, Centre for Clinical Epidemiology, Jewish General Hospital, 3755 Côte \\ Ste-Catherine, H-461, Montréal, Québec, Canada H3T 1E2. E-mail: samy.suissalamcgill.ca
}

@ERSpublications

Adding 2nd long-acting bronchodilator in COPD safe for cardiovascular events; heart failure risk slightly increased http://ow.ly/Yj3G309ReeN

Cite this article as: Suissa S, Dell'Aniello S, Ernst P. Concurrent use of long-acting bronchodilators in COPD and the risk of adverse cardiovascular events. Eur Respir J 2017; 49: 1602245 [https://doi.org/ $10.1183 / 13993003.02245-2016]$.

ABSTRACT The cardiovascular risk of concurrently using long-acting $\beta_{2}$-agonists (LABAs) and anticholinergics (LAMAs) in COPD is uncertain. We assessed the comparative cardiovascular and cerebrovascular safety of adding a second long-acting bronchodilator in patients with COPD.

We identified a cohort of COPD patients, new users of LABA or the LAMA tiotropium during 2002-2012, from the UK Clinical Practice Research Datalink. Using high-dimensional propensity scores, each patient adding a second bronchodilator was matched with a patient who remained on monotherapy. Patients were followed for 1 year for the occurrence of acute myocardial infarction (AMI), stroke, heart failure and arrhythmia.

The cohorts included up to 31174 patients adding a bronchodilator matched to 31174 patients remaining on bronchodilator monotherapy. Adding a long-acting bronchodilator, compared to remaining on monotherapy, was not associated with an increased risk of AMI (hazard ratio (HR) 1.12, 95\% CI 0.92-1.36), stroke (HR 0.87, 95\% CI 0.69-1.10) or arrhythmia (HR 1.05, 95\% CI 0.81-1.36), but the risk was elevated for heart failure (HR 1.16, 95\% CI 1.03-1.30).

Adding a second long-acting bronchodilator in the real-world-setting treatment of COPD does not increase the risk of most cardiovascular events. The modest increase for heart failure warrants further investigation.

This article has supplementary material available from erj.ersjournals.com

Received: Nov 142016 | Accepted after revision: Jan 092017

Support statement: This research was funded in part by grants from the Canadian Institutes of Health Research (CIHR), the Canadian Foundation for Innovation (CFI) and Boehringer Ingelheim. S. Suissa is the recipient of the James McGill Professorship award. The sponsors were not directly involved in the design and conduct of the study, interpretation of the data or preparation of the manuscript. Boehringer Ingelheim was invited to comment on the study protocol and the manuscript. Modifications resulting from comments were made independently by the authors on the basis of scientific and editorial merit. Funding information for this article has been deposited with the Crossref Funder Registry.

Conflict of interest: Disclosures can be found alongside this article at erj.ersjournals.com

Copyright OERS 2017 


\section{Introduction}

Long-acting bronchodilators, including long-acting $\beta_{2}$-agonists (LABAs) and anticholinergics (LAMAs) such as tiotropium, continue to be recommended as first-line maintenance therapy in the management of chronic obstructive pulmonary disease (COPD) [1-3]. These treatment guidelines also generally recommend the addition of another long-acting bronchodilator as a subsequent step in treatment when the disease is not controlled and, as a result, combined inhalers containing a LABA and a LAMA are now available for this second step in treatment. However, long-acting bronchodilators can potentially cause cardiac complications, including tachyarrhythmia and coronary insufficiency $[4,5]$.

Observational studies and randomised trials have reported some cardiovascular risks with these agents, although they only considered their effects in monotherapy [6-11]. However, with the expected increasing use of these agents in combination, the question of the potential cardiovascular risk arising from the concurrent use of two long-acting bronchodilators becomes pertinent and has yet to be studied. The TOnado trials, which compared a LABA-LAMA combination with its individual components, and the FLAME trial, which compared a LABA-LAMA combination with a LABA-inhaled corticosteroid (ICS), although large in terms of the numbers of subjects, were insufficiently sized to assess the risks of cardiovascular events (all less than $2 \%)[12,13]$. However, the FLAME trial reported a numerical imbalance in non-fatal acute myocardial infarction, with 11 events under LABA-LAMA versus five under LABA-ICS [13].

As these long-acting bronchodilators have been used for years to treat COPD, we assessed the comparative safety of adding a second long-acting bronchodilator (either adding a LABA to tiotropium use or, vice versa, adding tiotropium to LABA use) on the incidence of acute myocardial infarction (AMI), stroke, heart failure and arrhythmia in patients with COPD, relative to monotherapy.

\section{Methods}

Data source

We used the Clinical Practice Research Datalink (CPRD), a primary care database from the UK that contains primary care medical records for over 10 million people enrolled from over 600 practices. Participating general practitioners were trained to record medical information, including demographic data, lifestyle factors and medical diagnoses, using the Read classification. Prescriptions are automatically transcribed using the UK Prescription Pricing Authority Dictionary. The CPRD can be linked for approximately half of the practices to the Hospital Episodes Statistics (HES) database, which provides hospitalisation information. The recorded information on drug exposures and diagnoses was validated and proven to be of high quality [14-16].

\section{Study design}

We first formed a base cohort of all new users of long-acting bronchodilators, either a LABA or tiotropium, from January 1, 2001 until August 31, 2012, with new use defined as a first prescription of either drug with none during the previous 2 years. Thus, all patients in this base cohort were required to have at least 2 years of up-to-standard medical history prior to the first cohort-defining prescription to allow the identification of new use. Patients initiating treatment with both bronchodilators on the same date were excluded. To increase the likelihood of a diagnosis of COPD, we only included patients who were 55 years of age or more on the date of this initial prescription. This also increased the representativeness of the study by including as many patients as possible, although we also performed an analysis using the $90 \%$ of patients who had a COPD diagnosis prior to or at the time of the initial long-acting bronchodilator.

The study cohorts were formed by identifying all subjects from the base cohort who subsequently received the other long-acting bronchodilator class as a second additional bronchodilator, defined as both bronchodilator prescriptions given simultaneously on the same day. To avoid confounding issues related to more severe patients being the first ones to be prescribed the newer bronchodilator on the market, in this case tiotropium, we only considered subjects who added the other bronchodilator at least 1 year after tiotropium was first used, namely September 25, 2002.

For each subject who was on a LABA and subsequently added tiotropium, we identified a propensity score-matched reference subject who also was on a LABA but remained on a LABA only at the time the subject added the tiotropium prescription, using a prevalent new-user design [17]. The potential reference subjects were selected from the corresponding exposure sets, namely from the base cohort among all subjects who were already on a LABA, who received a LABA prescription within a month of the date tiotropium was added, and who were in the base cohort for the same duration ( \pm 3 months) as the combination-exposed subjects. Thus, the time span between base cohort entry (monotherapy initiation) and study cohort entry (addition of the second bronchodilator or matched continuation) was inherently a matching covariate. To allow matching on the propensity to add tiotropium, we used the high-dimensional propensity score (HDPS) technique by identifying all available data (e.g. diagnoses, procedures and medications) in the one-year 
period prior to the date of the matched set and applying conditional logistic regression to estimate a combination treatment propensity score [18]. In addition to the 500 variables selected by HDPS, the propensity score model forced age, sex, exacerbation in the previous 30 days and presence of the outcome in the 2 years prior to the date of the matched set. Then, for each combination-exposed subject and starting chronologically with the first, we identified one reference subject from the list of potential subjects at that time point, matched on prior ICS use and on the closest propensity score.

An identical approach was used for the subjects who initiated treatment on tiotropium and added a LABA, for whom matched subjects were taken from those who were only on tiotropium at that time. The two cohorts were pooled to form the final study cohort. Subjects were followed up for outcome events occurring during the 1-year span after cohort entry, namely the date of the matched set defined by either the addition of a second bronchodilator or the continuation of a single bronchodilator, with follow-up ending at the earliest of the date of cardiovascular outcome, 1 year after cohort entry, the date of death, August 31, 2013, or the end of coverage in the practice, whichever occurred first.

\section{Outcome events}

For myocardial infarction, heart failure and stroke, we identified all subjects with a Read code recorded in the general practitioner (GP)'s diagnostic field for these conditions occurring during the year after cohort entry. The diagnostic codes and algorithms to identify these outcomes have been shown to be highly valid for these outcomes and have been used in several studies using the CPRD [19-25]. In particular, for heart failure, probable cases were defined as a diagnosis of heart failure with either death within 30 days or treatment with at least two medications among the classes ACE/ARB (angiotensin-converting enzyme inhibitors and angiotensin receptor blockers), diuretics and digitalis, whereas possible cases were defined as diagnosis of heart failure and either treatment with one or more medications among the ACE/ARB, diuretics and digitalis classes, or death between 30 and 90 days after diagnosis. For the outcome of arrhythmia, which cannot be based strictly on GP diagnoses, we used the subcohort of the study cohort members that can be linked to the HES database. Thus, arrhythmia was based on hospitalisation diagnoses with ICD-10 codes I46.x-I49.x, R00.0 and R00.1. These definitions from hospitalised data have been used in several studies involving COPD [26, 27].

\section{Covariates}

As the HDPS approach identifies the 500 most likely confounders, we list here the covariates of particular interest that we report and compare with respect to the balance between the two groups. These include age, sex, body mass index (BMI), smoking status and excessive alcohol consumption. Baseline comorbidity, measured in the baseline year prior to study cohort entry, used diagnoses for arrhythmia, cardiomegaly, atherosclerosis, anaemia, hypertension, congenital structural cardiovascular abnormalities, diabetes, thyroid disease, hyperlipidaemia, renal failure and pneumonia, as well as presence of the study outcomes. Additionally, all prescriptions for angiotensin-converting enzyme inhibitors, angiotensin receptor blockers, $\beta$-blockers, thiazide diuretics, loop diuretics, antiarrhythmics, digoxin (digitalis), nitrates, statins, clopidogrel, aspirin, insulin, oral hypoglycaemics, nonsteroidal anti-inflammatory drugs, opioid analgesics and acetaminophen given during the 1 year baseline period prior to study cohort entry were identified. In addition, prescriptions for drugs hypothesised to lengthen the QT interval, such as macrolides, antidepressants, cisapride, antipsychotics and so on, were identified [28].

Baseline COPD severity was measured by the number of prescriptions for short-acting $\beta$-agonists and anticholinergics, methylxanthines, as well as inhaled corticosteroids (whether alone or combined with the LABA) and oral corticosteroids used during the baseline year prior to study cohort entry. Baseline inhaled corticosteroid use was defined prior to the day of study cohort entry and thus did not include the LABA prescription used to define cohort entry, which may be combined with an inhaled corticosteroid. A new prescription for prednisolone or a physician diagnosis in the 30 days prior to study cohort entry was used to identify recent COPD exacerbations [29].

\section{Data analysis}

The crude 1 year cumulative incidence and rate of the outcomes was estimated for the two long-acting bronchodilator groups. The comparative analyses used a time-dependent Cox proportional hazard regression model to perform an as-treated analysis that assesses the effect of current use of the combination or monotherapy exposure on the outcome occurrence. Thus, for the as-treated analysis, patients who switched to the other group during follow-up were censored at that time. Current use was defined as exposure to the initial combination or monotherapy within the 60 day period of the date defined by the risk set of the Cox model. This model included patient characteristics such as age in 10 year categories and sex, as well as the decile of propensity score for each patient obtained from the HDPS model for matching purposes and year of cohort entry. We also adjusted for the use of ipratropium 
in the year preceding study cohort entry, because an imbalance was observed after propensity score matching and we adjusted for the time since initiation of treatment with the long-acting bronchodilator, even if this was a matching factor because the matching was done within \pm 3 months.

Several sensitivity analyses were performed. First, to assess potential reverse causality bias from the as-treated analyses, we also used an intention-to-treat (ITT) approach, which compares combination to monotherapy with respect to the outcome occurrence in the subsequent year, irrespective of changes in treatment during this time, using a time-fixed Cox proportional hazard regression model. Second, analyses were stratified by the order that the drug was added, namely adding tiotropium to the initiated LABA and vice versa, a recorded diagnosis of COPD, prior use of ICS and by the presence of a recent COPD exacerbation in the 30 days prior to study cohort entry. Third, analyses were restricted to subjects with no presence of the outcome at baseline and repeated using the probable heart failure definition only. Fourth, the 60 day period used to define current use in the as-treated analyses was repeated with 30 and 90 day periods. Also, to verify the potential protopathic bias [30] from the possibility that a very recent prescription for a bronchodilator may have occurred in relation to dyspnoea related to early stages of myocardial dysfunction rather than a worsening of COPD, we repeated the as-treated analysis after eliminating events occurring in the first 30 and 90 days of follow-up. We also repeated the analyses allowing exposure to the combination during follow-up to be defined as two prescriptions within \pm 3 days of each other. Lastly, analyses were repeated with a maximum difference in propensity score distance of 0.05. All analyses were conducted using SAS version 9.4.

The study protocol was approved by the Independent Scientific Advisory Committee of the CPRD (Protocol 13_093RA) and the Ethics Committee of the Jewish General Hospital (JGH Protocol \#13-096), Montreal, QC, Canada.

\section{Results}

We identified 463899 patients with a prescription for LABA or tiotropium between January 1, 2001 and August 31, 2013, from which there remained 132245 eligible subjects forming the base cohort of initiators of a long-acting bronchodilator, including 80948 who formed the base subcohort linked to the HES database (figure 1). There were 32741 patients who received a LABA and tiotropium prescription on the same day during follow-up, including 16,814 who were on a LABA and added tiotropium and 15927 who were on tiotropium and added a LABA (figure 1). For the base subcohort linked to the HES, 19936 patients received a LABA and tiotropium on the same day during follow-up, including 10263 who were on a LABA and added tiotropium and 9673 who were on tiotropium and added a LABA.

Some of the patients adding another long-acting bronchodilator could not be matched. For instance, for the analysis of the AMI outcome, of the 32741 patients entering the base cohort on tiotropium and adding a LABA, 1603 (5\%) could not be matched. The main reasons for not finding a match for these patients were the requirement for matching on prior ICS use and the diminishing pool of monotherapy users eligible for the patients adding later in study period.

The baseline characteristics of the matched cohorts are shown in table 1 for the AMI and arrhythmia outcomes. Corresponding tables for the stroke and heart failure outcomes, shown in the supplementary material, are practically identical to table 1 due to the trivial variations in HDPS matching for each outcome. After propensity score matching, the patients adding the other bronchodilator have a similar baseline profile to those remaining on monotherapy, with the exception of prior ipratropium bromide use, for which statistical adjustment was added to the model.

The as-treated rates of outcome per 100 per year in the reference monotherapy groups were 1.0 for AMI, 0.8 for stroke, 2.9 for heart failure and 1.0 for arrhythmia (table 2). Table 2 presents the hazard ratio of each outcome associated with adding a long-acting bronchodilator to the previous one (combination), relative to remaining on a single long-acting bronchodilator (monotherapy), from the as-treated analysis. For AMI, the hazard ratio was 1.12 (95\% CI 0.92-1.37), while for stroke it was 0.87 (95\% CI 0.69-1.10) and for arrhythmia 1.05 (95\% CI 0.81-1.36). The risk of heart failure (HR 1.16, 95\% CI 1.03-1.30) was increased when adding a bronchodilator.

The results of the ITT sensitivity analysis shown in table 3 are similar to the results for the as-treated analysis. The other sensitivity analyses did not generally alter the results. These sensitivity analyses are presented in table 4 for the heart failure outcome because of its overall statistical significance.

\section{Discussion}

In this observational study based on real-world data, we found that adding a second long-acting bronchodilator, either the anticholinergic tiotropium to a LABA or vice versa, results in comparable 1 year cardiovascular risks of AMI, stroke and arrhythmia, relative to remaining on long-acting bronchodilator 


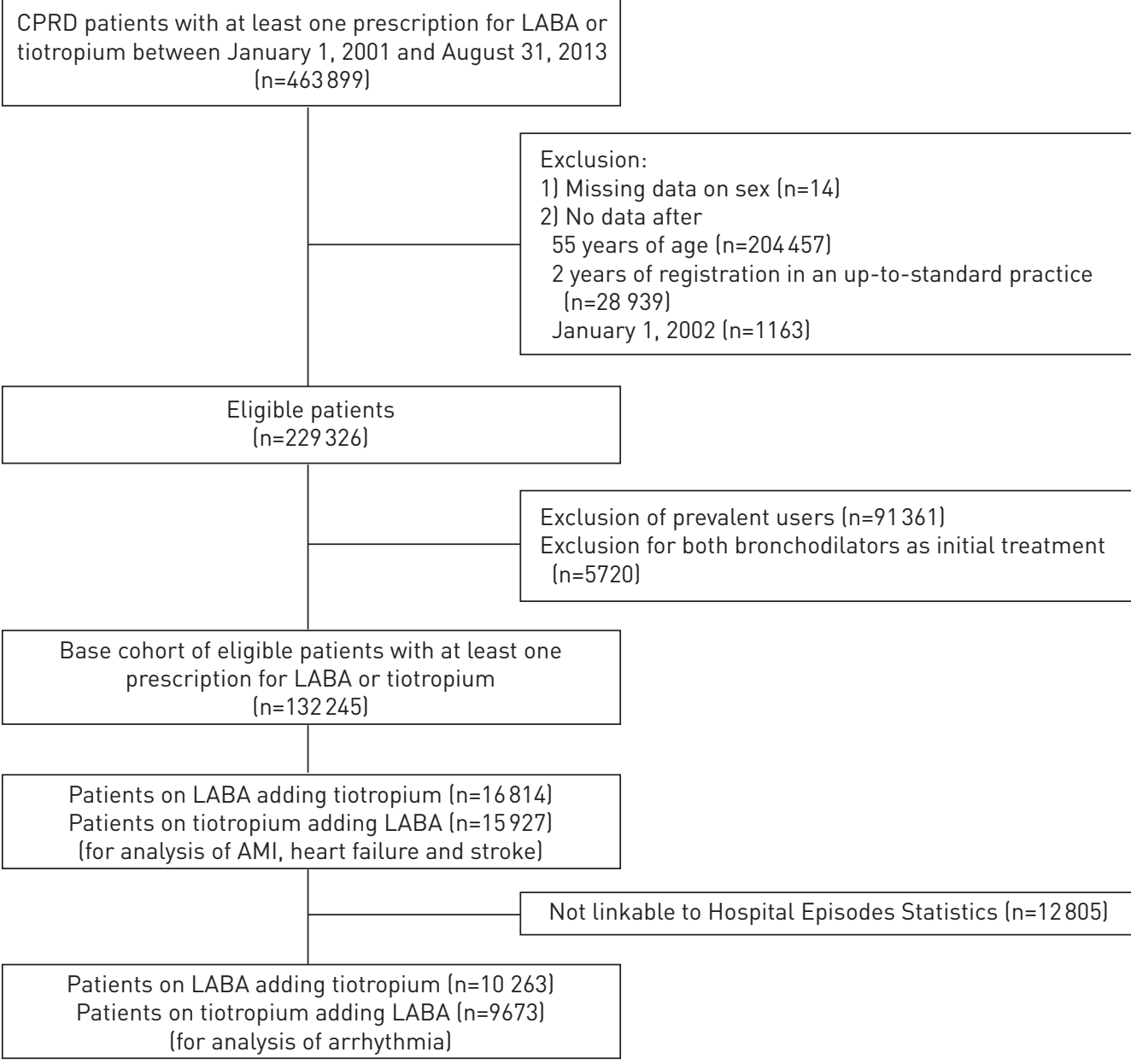

FIGURE 1 Flow chart of study cohort formation. LABA: long-acting $\beta_{2}$-agonists; CPRD: Clinical Practice Research Datalink; AMI: acute myocardial infarction.

monotherapy. However, the incidence of heart failure was marginally higher for those who added a second long-acting bronchodilator.

This is the first study to evaluate the cardiovascular safety of the concurrent use of long-acting bronchodilators in COPD, which is of particular relevance because these agents have been suspected to cause cardiac complications [5]. The long-acting anticholinergics are believed to suppress parasympathetic control and LABAs stimulate sympathetic tone, possibly leading to tachyarrhythmia and coronary insufficiency $[4,26,27]$. A pooled safety analysis of the tiotropium trials reported a decreased risk of cardiac failure (rate ratio (RR) $0.81,95 \%$ CI $0.69-0.96$ ) and of myocardial infarction (RR $0.85,95 \%$ CI $0.67-1.09$ ) compared with placebo [31]. However, this pooled analysis did not provide a stratified analysis by concurrent use of a LABA (over $37 \%$ of all patients) to permit an assessment of the effect of adding tiotropium to a LABA on these outcome events. The recent relatively large trials of the effectiveness of LABA-LAMA combinations, despite each studying close to 2000 patients treated on a LABA-LAMA combination, were insufficiently sized to assess the risks of these relatively infrequent cardiovascular events $[12,13]$. Indeed, the pooled TOnado trials, which compared the combination of tiotropium+olodaterol with the individual components, reported rate ratios of major adverse cardiac events of 1.07 (95\% CI 0.66-1.73) and 1.11 (95\% CI $0.68-1.80$ ) on comparing the combination with olodaterol and tiotropium monotherapies, respectively [12]. The upper limit of these confidence intervals does not rule out important increases in risk. The FLAME trial, which compared the indacaterol-glycopyrronium combination with salmeterol-fluticasone, found (our calculation) a rate ratio of major adverse cardiac events of 1.14 (95\% CI 0.64-2.05), which also does not rule out important increases in risk [13]. Moreover, the FLAME trial reported a numerical imbalance in non-fatal AMI, with 11 events under indacaterol-glycopyrronium versus five under salmeterolfluticasone [13]. With its much larger size, our study provides accurate cardiovascular and cerebrovascular risk estimates over the first year after adding a second long-acting bronchodilator. In particular, we did not find a higher incidence of AMI when adding a second long-acting bronchodilator. 
TABLE 1 Baseline characteristics of the cohort of 31138 patients who added tiotropium to a long-acting $\beta_{2}$-agonist (LABA) or vice versa (combination) and their propensity score and prior inhaled corticosteroid-matched 31138 users of a LABA or tiotropium who did not add (monotherapy), used for analysis of acute myocardial infarction (AMI) outcome, and for the subset of 18861 combination users and their matched 18861 monotherapy users selected from the subcohort of Hospital Episodes Statistics (HES) database-linked data, for arrhythmia outcome

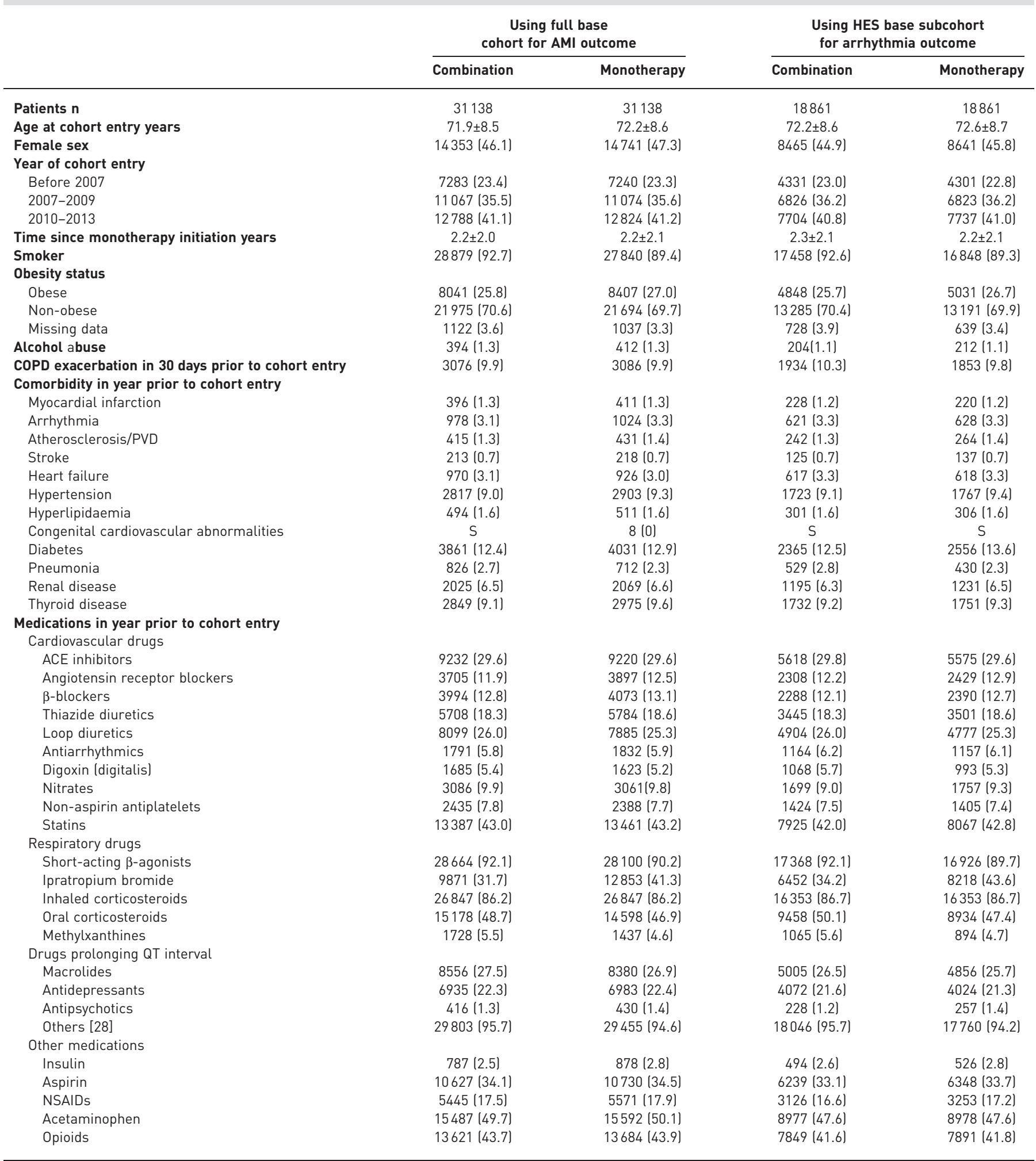

Data are presented as mean \pm SD or $n$ (\%). COPD: chronic obstructive pulmonary disease; PVD: peripheral vascular disease; ACE: angiotensin-converting enzyme; NSAID: nonsteroidal anti-inflammatory drug; S: small cells (count $\leqslant 5$ ) were suppressed due to privacy restriction. 
TABLE 2 Crude and adjusted hazard ratios of cardiovascular outcomes associated with adding a second long-acting bronchodilator (combination) compared with remaining on a single long-acting bronchodilator (monotherapy) in patients with chronic obstructive pulmonary disease, with 1 year follow-up, from the as-treated analysis

\section{Patients n Events n Person-years Rate per 1000 per year Crude ${ }^{\#}$ HR Adjusted ${ }^{\text {I }}$ HR $(95 \%$ CI)}

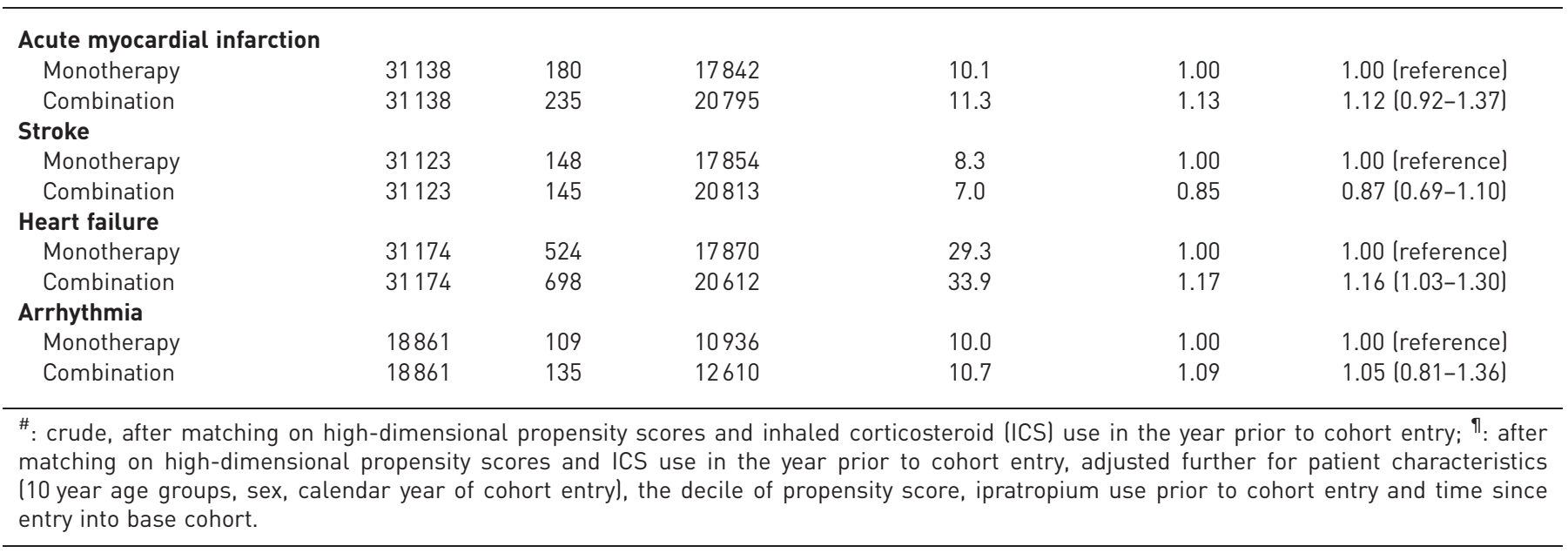

The statistically significant $16 \%$ increase in the risk of heart failure with the addition of a second long-acting bronchodilator is robust when subjected to multiple sensitivity analyses. These analyses suggest that these excess events of heart failure are not occurring early after adding the second bronchodilator; a potential source of protopathic bias from a recent prescription for a bronchodilator given for dyspnoea related to early stages of myocardial dysfunction rather than a worsening of COPD [30]. Indeed, ITT analysis of the overall cohort also produced a similar hazard ratio of heart failure of 1.14 (95\% CI 1.03 1.26) with the addition of the second bronchodilator, while the analysis based on removing the heart failure events that occurred early in the first 30 or 90 days after adding the second bronchodilator resulted in hazard ratios of 1.22 (95\% CI 1.07-1.39) and 1.28 (95\% CI 1.09-1.51), respectively. The potential for bias due to a prior heart failure diagnosis with continuing COPD treatment can be ruled out by the hazard ratio of 1.21 (95\% CI 1.05-1.39) among patients with no prior heart failure. If this risk is real and not due to residual confounding, the $16 \%$ increase translates to 4.6 additional cases of heart failure per year for every 1000 patients adding a second bronchodilator.

A major strength of the study is its large size, comprising over 30000 patients who added a second longacting bronchodilator, thus allowing more precise estimates than the recent trials $[12,13]$. In addition, our

TABLE 3 Crude and adjusted hazard ratios of cardiovascular outcomes associated with adding a second long-acting bronchodilator (tiotropium or long-acting $\beta_{2}$-agonist) compared with remaining on a single long-acting bronchodilator in patients with chronic obstructive pulmonary disease, with 1 year follow-up, from the intent-to-treat analysis

\section{Patients n Events n Person-years Rate per 1000 per year Crude ${ }^{\#}$ HR Adjusted" HR $(95 \% \mathrm{Cl})$}

\begin{tabular}{|c|c|c|c|c|c|c|}
\hline \multicolumn{7}{|c|}{ Acute myocardial infarction } \\
\hline Monotherapy & 31138 & 251 & 23253 & 10.8 & 1.00 & 1.00 (reference) \\
\hline Combination & 31138 & 318 & 27956 & 11.4 & 1.07 & $1.06(0.89-1.25)$ \\
\hline \multicolumn{7}{|l|}{ Stroke } \\
\hline Combination & 31123 & 213 & 27980 & 7.6 & 0.92 & $0.94(0.77-1.15)$ \\
\hline \multicolumn{7}{|l|}{ Heart failure } \\
\hline Monotherapy & 31174 & 697 & 23192 & 30.0 & 1.00 & 1.00 (reference) \\
\hline Monotherapy & 18861 & 149 & 14076 & 10.6 & 1.00 & 1.00 (reference) \\
\hline Combination & 18861 & 184 & 16919 & 10.9 & 1.04 & $1.01(0.81-1.26)$ \\
\hline
\end{tabular}

\#: crude, after matching on high-dimensional propensity scores and inhaled corticosteroid (ICS) use in the year prior to cohort entry; ${ }^{\natural}$ : after matching on high-dimensional propensity scores and ICS use in the year prior to cohort entry, adjusted further for patient characteristics (10 year age groups, sex, calendar year of cohort entry) as well as the decile of propensity score, ipratropium use prior to cohort entry and time since entry into base cohort. 
TABLE 4 As-treated sensitivity analyses for the heart failure outcome associated with adding a second long-acting bronchodilator (combination) compared with remaining on a single long-acting bronchodilator (monotherapy) in patients with chronic obstructive pulmonary disease (COPD), with 1 year follow-up

\begin{tabular}{|c|c|c|c|c|c|c|}
\hline & Patients n & Events $\mathrm{n}$ & Person-years & $\begin{array}{l}\text { Rate per } 1000 \\
\text { per year }\end{array}$ & Crude $^{\#}$ HR & $\begin{array}{l}\text { Adjusted" } \\
\text { HR }(95 \% \text { CI) }\end{array}$ \\
\hline \multicolumn{7}{|l|}{ Initiating with a LABA } \\
\hline Adding tiotropium & 16814 & 380 & 11002 & 34.5 & 1.10 & $1.11(0.95-1.28)$ \\
\hline \multicolumn{7}{|l|}{ Initiating on tiotropium } \\
\hline Tiotropium monotherapy & 14360 & 187 & 7089 & 26.4 & 1.00 & 1.00 (reference) \\
\hline Adding a LABA & 14360 & 318 & 9610 & 33.1 & 1.30 & $1.28(1.07-1.54)$ \\
\hline Combination & 2628 & 73 & 1528 & 47.8 & 1.34 & $1.18(0.86-1.62)$ \\
\hline \multicolumn{7}{|l|}{ Recorded COPD diagnosis } \\
\hline Monotherapy & 27069 & 434 & 15306 & 28.4 & 1.00 & 1.00 (reference) \\
\hline Combination & 28546 & 625 & 19084 & 32.8 & 1.18 & $1.18(1.04-1.33)$ \\
\hline \multicolumn{7}{|l|}{ No recent COPD exacerbation } \\
\hline Monotherapy & 28126 & 466 & 16286 & 28.6 & 1.00 & 1.00 (reference) \\
\hline \multicolumn{7}{|l|}{ No baseline ICS use } \\
\hline Monotherapy & 4291 & 89 & 2784 & 32.0 & 1.00 & 1.00 (reference) \\
\hline Combination & 4291 & 97 & 2685 & 36.1 & 1.11 & $1.13(0.84-1.50)$ \\
\hline \multicolumn{7}{|l|}{ Baseline ICS use } \\
\hline Monotherapy & 26883 & 435 & 15086 & 28.8 & 1.00 & 1.00 (reference) \\
\hline Combination & 26883 & 601 & 17926 & 33.5 & 1.19 & $1.17(1.03-1.32)$ \\
\hline \multicolumn{7}{|c|}{ Current use defined within a 30 -day period } \\
\hline Monotherapy & 31174 & 406 & 13640 & 29.8 & 1.00 & 1.00 (reference) \\
\hline Combination & 31174 & 481 & 14504 & 33.2 & 1.12 & $1.11(0.97-1.27)$ \\
\hline \multicolumn{7}{|c|}{ Current use defined within a 90-day period } \\
\hline Monotherapy & 31174 & 564 & 19212 & 29.4 & 1.00 & 1.00 (reference) \\
\hline Combination & 31174 & 793 & 23036 & 34.4 & 1.19 & $1.18(1.05-1.31)$ \\
\hline \multicolumn{7}{|c|}{ Using probable heart failure outcome definition } \\
\hline Monotherapy & 29392 & 399 & 15466 & 25.8 & 1.00 & 1.00 (reference) \\
\hline Combination & 30589 & 567 & 18073 & 31.4 & 1.23 & $1.22(1.07-1.39)$ \\
\hline \multicolumn{7}{|c|}{$\begin{array}{l}\text { Eliminating outcome events in first } 90 \text { days of } \\
\text { follow-up }\end{array}$} \\
\hline Monotherapy & 25879 & 255 & 11786 & 21.6 & 1.00 & 1.00 (reference) \\
\hline Combination & 29325 & 380 & 13826 & 27.5 & 1.28 & $1.28(1.09-1.51)$ \\
\hline \multicolumn{7}{|c|}{ No heart failure diagnosis before cohort entry } \\
\hline Monotherapy & 29763 & 340 & 17198 & 19.8 & 1.00 & 1.00 (reference) \\
\hline Combination & 29737 & 474 & 19821 & 23.9 & 1.23 & $1.21(1.05-1.39)$ \\
\hline
\end{tabular}

LABA: long-acting $\beta_{2}$-agonists; ICS: inhaled corticosteroid; PS: propensity score. ${ }^{\#}$ : crude, after matching on high-dimensional propensity scores and ICS use in the year prior to cohort entry; ": after matching on high-dimensional propensity scores and ICS use in the year prior to cohort entry, adjusted further for patient characteristics (10 year age groups, sex, calendar year of cohort entry), as well as the decile of propensity score, ipratropium use prior to cohort entry and time since entry into base cohort.

study included patients from the time of their addition of the second long-acting bronchodilator, which avoids bias from depletion of susceptible, which can be problematic for safety studies [32]. Indeed, adverse effects such as AMI and arrhythmia can occur with initiation of these therapies, but trials to date, such as FLAME, included patients who at baseline were already using LAMAs (61\%) or LABAs (67\%), some of 
whom were probably using both, so patients who had experienced early effects of these drugs were probably not included in the trial [13].

Our study has some limitations. Exposure to long-acting bronchodilators was measured from prescriptions, introducing potential exposure misclassification from uncertainty about the drugs actually being taken. However, the outcome events used validated definitions and are thus less subject to misclassification. Our definition of COPD was based exclusively on age and medication to increase the representativeness of the study by including as many patients as possible in assessing these safety questions. Nevertheless, the results remained identical in the analysis using the $90 \%$ of patients who had a recorded COPD diagnosis. In addition, the study probably captured patients who also have asthma, which should have been balanced in the two comparison groups by the use of the propensity scores. Nevertheless, despite the use of high-dimensional propensity scores, some residual confounding may be present. Moreover, in our analysis we did not consider the changes in the confounding factors such as use of cardiovascular drugs during follow-up, although the 1 year follow-up period is probably too short to drastically change the risk profile of the patients from the baseline period and to change it differentially between the two groups.

Overall, in this real-world setting study of the treatment of COPD, the addition of a second long-acting bronchodilator as recommended by COPD treatment guidelines when the disease worsens, appears safe with respect to cardiovascular and cerebrovascular risks, except perhaps for a small increase in the risk of heart failure, which warrants continued monitoring.

\section{Acknowledgements}

All authors participated in the study design, data interpretation and writing of the manuscript. S. Suissa acquired the data and acts as guarantor of the manuscript.

\section{References}

1 Pauwels RA, Buist AS, Calverley PM, et al. Global strategy for the diagnosis, management, and prevention of chronic obstructive pulmonary disease. NHLBI/WHO Global Initiative for Chronic Obstructive Lung Disease (GOLD) Workshop summary. Am J Respir Crit Care Med 2001; 163: 1256-1276.

2 Rabe KF, Hurd S, Anzueto A, et al. Global strategy for the diagnosis, management, and prevention of chronic obstructive pulmonary disease: GOLD executive summary. Am J Respir Crit Care Med 2007; 176: 532-555.

3 Vestbo J, Hurd SS, Agusti AG, et al. Global strategy for the diagnosis, management, and prevention of chronic obstructive pulmonary disease: GOLD executive summary. Am J Respir Crit Care Med 2013; 187: 347-365.

4 Singh S, Loke YK, Enright P, et al. Pro-arrhythmic and pro-ischaemic effects of inhaled anticholinergic medications. Thorax 2013; 68: 114-116.

5 Lahousse L, Verhamme KM, Stricker BH, et al. Cardiac effects of current treatments of chronic obstructive pulmonary disease. Lancet Respir Med 2016; 4: 149-164.

6 Jara M, Wentworth C III, Lanes S. A new user cohort study comparing the safety of long-acting inhaled bronchodilators in COPD. BMJ Open 2012; 2: e000841.

7 Gershon A, Croxford R, Calzavara A, et al. Cardiovascular safety of inhaled long-acting bronchodilators in individuals with chronic obstructive pulmonary disease. JAMA Intern Med 2013; 173: 1175-1185.

8 Vogelmeier C, Hederer B, Glaab T, et al. Tiotropium versus salmeterol for the prevention of exacerbations of COPD. N Engl J Med 2011; 364: 1093-1103.

9 Rodrigo GJ, Neffen H. Comparison of indacaterol with tiotropium or twice-daily long-acting beta-agonists for stable COPD: a systematic review. Chest 2012; 142: 1104-1110.

10 Decramer ML, Chapman KR, Dahl R, et al. Once-daily indacaterol versus tiotropium for patients with severe chronic obstructive pulmonary disease (INVIGORATE): a randomised, blinded, parallel-group study. Lancet Respir Med 2013; 1: 524-533.

11 Suissa S, Dellaniello S, Ernst P. Long-acting bronchodilator initiation in COPD and the risk of adverse cardiopulmonary events: a population-based comparative safety study. Chest 2017; 151: 60-67.

12 Buhl R, Maltais F, Abrahams R, et al. Tiotropium and olodaterol fixed-dose combination versus mono-components in COPD (GOLD 2-4). Eur Respir J 2015; 45: 969-979.

13 Wedzicha JA, Banerji D, Chapman KR, et al. Indacaterol-glycopyrronium versus salmeterol-fluticasone for COPD. N Engl J Med 2016; 374: 2222-2234.

14 Jick SS, Kaye JA, Vasilakis-Scaramozza C, et al. Validity of the general practice research database. Pharmacotherapy 2003; 23: 686-689.

15 Herrett E, Thomas SL, Schoonen WM, et al. Validation and validity of diagnoses in the General Practice Research Database: a systematic review. Br J Clin Pharmacol 2010; 69: 4-14.

16 Herrett E, Gallagher AM, Bhaskaran K, et al. Data Resource Profile: Clinical Practice Research Datalink (CPRD). Int J Epidemiol 2015; 44: 827-836.

17 Suissa S, Moodie EE, Dell'Aniello S. Prevalent new-user cohort designs for comparative drug effect studies by time-conditional propensity scores. Pharmacoepidemiol Drug Saf 2016; doi: 10.1002/pds.4107.

18 Schneeweiss S, Rassen JA, Glynn RJ, et al. High-dimensional propensity score adjustment in studies of treatment effects using health care claims data. Epidemiology 2009; 20: 512-522.

19 Hammad TA, McAdams MA, Feight A, et al. Determining the predictive value of Read/OXMIS codes to identify incident acute myocardial infarction in the General Practice Research Database. Pharmacoepidemiol Drug Saf 2008; 17: 1197-1201.

20 Andersohn F, Suissa S, Garbe E. Use of first- and second-generation cyclooxygenase-2-selective nonsteroidal antiinflammatory drugs and risk of acute myocardial infarction. Circulation 2006; 113: 1950-1957. 
21 Renoux C, Dell'Aniello S, Garbe E, et al. Transdermal and oral hormone replacement therapy and the risk of stroke: a nested case-control study. Br Med J 2010; 340: c2519.

22 Renoux C, Dell'Aniello S, Brophy JM, et al. Dopamine agonist use and the risk of heart failure. Pharmacoepidemiol Drug Saf 2012; 21: 34-41.

23 Filion KB, Joseph L, Boivin JF, et al. Thiazolidinediones and the risk of incident congestive heart failure among patients with type 2 diabetes mellitus. Pharmacoepidemiol Drug Saf 2011; 20: 785-796.

24 Khan NF, Harrison SE, Rose PW. Validity of diagnostic coding within the General Practice Research Database: a systematic review. Br J Gen Pract 2010; 60: e128-e136.

25 Hall GC, Brown MM, Mo J, et al. Triptans in migraine: the risks of stroke, cardiovascular disease, and death in practice. Neurology 2004; 62: 563-568.

26 Wilchesky M, Ernst P, Brophy JM, et al. Bronchodilator use and the risk of arrhythmia in COPD: Part 1: Saskatchewan Cohort Study. Chest 2012; 142: 298-304.

27 Wilchesky M, Ernst P, Brophy JM, et al. Bronchodilator use and the risk of arrhythmia in COPD: Part 2: Reassessment in the Larger Quebec Cohort. Chest 2012; 142: 305-311.

28 Resources for professionals: QT drug lists by risk group. https://crediblemeds.org/new-drug-list Date last accessed: March 14, 2017.

29 Rodriguez-Roisin R. COPD exacerbations. 5: Management. Thorax 2006; 61: 535-544.

30 Horwitz RI, Feinstein AR. The problem of "protopathic bias" in case-control studies. Am J Med 1980; 68: 255-258.

31 Halpin DM, Dahl R, Hallmann C, et al. Tiotropium HandiHaler ${ }^{\circledR}$ and Respimat ${ }^{\oplus}$ in COPD: a pooled safety analysis. Int J Chron Obstruct Pulmon Dis 2015; 10: 239-259.

32 Miettinen OS, Caro JJ. Principles of nonexperimental assessment of excess risk, with special reference to adverse drug reactions. J Clin Epidemiol 1989; 42: 325-331. 